\title{
Enfermedades respiratorias crónicas y recurrentes de la infancia y la niñez en un hospital general
}

\author{
Patricia Cabezas B. '; Carłos Toro A. ${ }^{2}$; Maráa L. Boza C.'
}

\begin{abstract}
Resumen
Objetivo: Describir las causas más trecuentes de hospitalización de niños con enfermedades respiralorias cróricas o con sintemas respirolorios recurrentes y hacer una aproximación a sus cosles. Diseño. anólisis retrospectivo de las fichas c'íricas de los 322 ingresos de pocientes menores de is años intemados por enfermedad respiratoria crónica o ś́ntonas respiratcrios recurrentes en un servicio de pediatría de un hospital docente de Santiago (Chile) el oño 1994. Pocientes y método: En un formulario preparado en un programa Epinto 5.0 se registraron los diagnósticos príncipal, derivados, csociados y de las complicaciones o secuelas tcodificados según la clasificación internacional de enfermedadesi, 'os exámenes de loboratorio realizados en cado casc. Los costes de hospitalizacion y exámenes se estimaron valorizándolos con el arancel del Fondo Nacicncl de Salud [Foncsa, Chilel. Resultodos: En 21,2\% de las admisiones por entermedades respiratcrios y $11,1 \%$ del lobal ae ingresos se frotabo de erírmedodes respiratorios crónicos o recu"entes. Más de dos tercios lenían afecciones recurrentes, un quinto enfermedod respiratoria crónica propiamente tol y los restantes eran pacientes con enfermedod de base no respiratoria (preferentemente neurológicol y afección broncopuimorar securdaric. Las causas preciominantes de la admisión fueron, en los ires grupos, descompensociones funcionales por neumonias o episodios de obstrucción bronquial intercurrertes. Los exánenes de laboratcrio tradicionales de estas hospitalizaciones costaron $15,3 \%$ del gosto ictal del Servicio de Fediatria por este concepto $\$ \$ 607535$ e-tre $\$ 22735330$, sin considerar $\$ 5338240$ adicionales en otro tipo de exámenes de apoyo. La permanencia en el hospilal fue $30 \%$ nós larga en estos riños que en el lotal de pacientes adrilidos a pedialría. Las hospitalizaciones más largas se registraron en los enfermos respirgtorios crónicos o con enfermedad de bose no respiratcria.
\end{abstract}

(Palabras clave: enfermedades respiratorios crónicas, recurrenles, causas de ingreso, coslos de hospitalización.)

\section{Infancy and childhood chronic and recurrent respiratory diseases at a Hospital Pediatric Service in Santiago, Chile}

Objective: to describe the most frequent causes of hospital admissiors of children with chronic respiratory diseoses or recurre-t respiratory symploms, and their economic impoct as compored to the whole pediatric admissions. Design: rerospective, descriplive, partial cost effectiveness analysis in a pediatric unir of a public, university associated, gereral hospital for middle 'ow income population at downtown Santiago, Chile. Palients: records of 242 hospitalizations to ' 43 palients among 322 admissions along year 1994 by chronic respiratory diseases or recurrent respiratory symptoms were available for review. These represented $21,2 \%$ of odmissions due to respiratory diseases, and $11,1 \%$ of oll pediatric admissions. Recurrent respiratory symptoms $(09,4 \%)$-mostly bronquial hyperreactivity syn-dromethronic respiratory diseases (19\%), and secondary respiratory diseases in patients whith non respiratory basal diseases (11,6\%)-mainly neurologic abnormalities-, were the most f-equent bosai diseoses, while the most frequent direct couses of admission in oll these patients were pneumonio and bronchial obstructive crisis. Chronic respiratory disease patients and palients with basal non respiralory diseoses accounted for the longer hospital stays. Averoge hospilal stay of chionic and recurreril respiracry disease patiens was 11.4 days, or $30 \%$ longer than the mean for the pedichric wards as a whole. Expenses in routine laboratery tests of this potients occounted for more, than one sixth of all laborctory expenses of the genera pedictric services iUS\$24020 out of US\$150 8381. Conclusions: patients with chrenic or recurrenl respiratory diseases generale more hospital expenses than general pediatric patients even if cost of diagrostic work.up is not considered. Many of these expenses may be lowered by appropriate coordination and equipement al amoulatory care levels.

[Koy words: respiratory diseases chronic, recurrence, aomission, nospitalization cost.]

1. Departamento de Pediatría. Cirugía Pediátrica y Ortopedia Centro, Facultad de Medicina, Universidad de Chile. Servicio de Pediatría. Unidad Respiratorio Infantil. Hospital Clínico San Borja-Arriarán.

2. Departamento de Pediatrfa, Cirugía Pediálrica y Ortopedia Centro, Facultad de Medicina, Universidad de Chile. 
Los avances en el diagnóstico y tratamiento de las enfermedades respiratorias en la infancia han aumentado la supervivencia y mejorado la calidad de vida de los pacientes con afecciones respiratorias crónicas y recurrentes. Esto se expresa en un aumento de la demanda por atenciones médicas ambulatorias y hospitalarias con el consiguiente incremento en el consumo de recursos.

No hay una definición universalmente aceptada de enfermedad respiratoria crónica y recuTente en la infancia ${ }^{1-5}$. Con frecuencia se considera que los síntomas respiratorios son crónicos si su duración se extiende por más de tres me$\operatorname{ses}^{1}$ y se usa la expresión "recurrente" para describir las afecciones que producen tres o más episodios de síntomas semejantes por año en el paciente, como ocurre en el síndrome bronquial obstructivo del lactante, secundario a infecciones virales, o a causas menos frecuentes como asma y otras enfermedades respiratorias cróni$\operatorname{cas}^{6,7}$

Los pacientes con enfermedades respiratorias crónicas o recurrentes demandan frecuentemente atenciones de salud de los niveles secundario y terciario, pero sus características -desde la perspectiva de la gestión de servicios hospita. larios de salud - no han sido suficientemente analizadas en el país. Con el propósito de contribuir, en una primera aproximación, con información útil para planificar la atención de los niños con estas afecciones en servicios del nivel terciario se revisaron retrospectivamente y describieron algunas características clínicas y evolutivas que parecían relevantes en los pacientes que ingresaron por esta causa a un servicio de pediatría típico de un hospital general metropolitano, durante un año, así como el consumo de ciertos recursos de diagnóstico y tratamiento que originaron.

\section{Material y Método}

Se revisaron las fichas clinicas de los pacientes que ingresaron a tas dependencias del Servicio de Pediatría del Hospital Clínico San Borja-Arriarán (HCSBA) en el año 1994 con enfermedades respiratorias crónicas o recurrentes. Estas fueron definidas de manera abitraria como aquellas cuyas principales manifestaciones tuviesen tres meses o thás de duración o tuviesen síntomas que se expresasen en tres o más episodios de rengudizaciones al año respectivamente. En un protocolo especialmente preparado para este propósito, se registraron los diagnósticos principales y secundarios, las caracteríslicas más destacadas de la enfermedad, la frecuencia de hospitalización por diagnósticos, las causas de ingreso, las complicaciones y tipo de recursos requeridos para su estudio y tratamiento y se realizó una estimación arbitraria sobre el gasto que generan en tipo y númeró de exámenes de laboratorio, tratamientos y días de hospitalización.

Los casos se ubicanon tevisando los libros de alta de cada unidad del Servicio de Pediatría, escogiendo entre los diagnósticos de egreso aquellos que correspondian a las definiciones de enfermedades respiratorias crónicas o recurrentes empleadas. En unas pocas situaciones de duda se revisaron, ademàs, las fichas clínicas del consultorio de atencion primaria de origen. La informacion fue obtenida de manera retrospectiva directamente de las observaciones clinicas y transcrita en un formulario precodificado preparado en un programa Epiinfo 5.0. por el autor principal. A los diagnósticos anotados en cada ingreso en los resúmenes de alta se asignt 6 ed código que correspondia de acuerdo con la clasificación internacional de enfermedades del año 1975, por ser el que estaba en uso en el servicio de estadísticas del hospital. En caso de registrarse diagnósticos no incluidos en dicha stasificación, se aplicó el código más cercano a la definición conceptual del problema, al que se agreg6 un número iđentificador arbitrario para clasificarlo con mayor precisión y uniformidad. Los diagnósticos de cada registro clínico fueron estructurados en cuatro estratos o categorias: enfermedad respiratoria de base (primer diagnóstico o diagnostico principal). complicación causada por la enfermedad respiratoria de base (habitualmente consignado como segundo diagnóstica), causa de bospitalización o descompensación y, finalmente, enfermedades no respiratorias (extrarrespiratorias) relevantes.

Durante el año 1994, en el Servicio de Pediatría del HCSBA, ocurrieron 2896 hospitalizaciones. de las cuales I $516(52.34 \%)$ cotrespondieron a enfermedades respiratorias y de estas $1194(78,8 \%)$ se clasificaron como agudas (episodios de 1 semana a 10 días de enfermedad, sislados, en pacientes sin antecedentes de enfermedad respiratoria cronica o recurrente). Otros $322(21,2 \%)$ ingresos fueron considerados debido a causas respiratorias crónicas o recumentes. De estas últimas, debido a dificultades para conseguir las historias clínicas dentro del periodo destinado para tal efecto. sólo fue posible completar la revisión de 242 (75.15\%), que constiłuyen el muterial de este análisis.

Por no haber estudios oficiales disponibles sobre los costos reales de los exámenes de laboratorio en el hospital encuestado, esos se estimaron sobre la buse del "Arancel $1^{*}$ del Fondo Nacional de Salud (FONASA 1) del ano 1994, lo que implica un importante sesgo, por cuanto su asignación suele basarse en una subestimación de los gastos reales implicitos.

\section{Resultados}

Los 242 ingresos por enfermedad respiratoria crónica y recurrente que fue posible revisar, involucraron a 143 pacientes (1,7 hospitalizaciones por paciente). Ciento cuarenta y cinco episodios $(59.9 \%)$ sucedieron en varones. La 
edad del conjunto oscilaba entre 7 días y 14 años (promedio 2 años, mediana de 12,84 me ses), en 176 casos ( $73 \%$ ) era inferior a 2 años y, en $134(55,4 \%)$ era menor de un año.

El diagnóstico principal o enfermedad de base más frecuentemente registrado fue "sindro. me bronquial obstructivo recurrente" ( $n=169$; $69,4 \%$ ), seguido por "neumopatías a repetición" $(n=28 ; 11,6 \%)$, ambos diagnósticos sindromáiicos, descriptivos, que nada dicen sobre la etiología de la enfermedad y en conjunto abarcan $81 \%$ del rubro. Así pues sólo en los restan. tes 46 ingresos $(19 \%)$ los diagnósticos principales corresponden a enfermedades respiratorias crónicas cuya etiologia o patogenia puede ser descrita con mayor precisión, como displasia broncopulmonar, asma bronquial, secuelas de infección por adenovirus, malformaciones broncopulmonares, fibrosis quística, hemosiderosis pulmonar, que consideradas en conjunto van en segundo lugar en el orden de frecuencia de hospitalización. Las causas de ingreso más frecuentes fueron neumonía y crjsis de obstrucción, con excepción de seis casos de malformaciones broncopulmonares y uno de bronquitis recurrente no obstructiva -en las cuales la razón del ingreso más frecuente fue el estudio de la enfermedad de base- $y$ algunos pacientes ingresados por complicaciones de la afección principal (tabla !).
Las complicaciones de la enfermedad de base se registraron en $35,2 \%$ de las 168 hospitalizaciones por síndrome bronquial obstructivo recurrente y consistieron en neumopatía reiterada en $44(26,2 \%)$, atelectasias segmentarias o lobares en 10 (6\%), bronquiectasias $4(2,4 \%)$ y daño pulmonar erónico en un paciente $(0,6 \%)$. Se registraron complicaciones en Ja mitad de los 28 casos en que el diagnóstico principal era neumonía a repetición, correspondiendo estas a bronquiectasias en $8(28,6 \%)$, insuficiencia respiratoria crónica con dependencia de oxígeno en $4(14,2 \%)$, daño pulmonar crónico no especificado en un caso $(3,6 \%)$ y síndrome bronquial obstructivo recurrente en otro. El segundo diagnostico en las hospitalizaciones por displasia broncopulmonar y fibrosis quística no correspondía a complicaciones de estas enfermedades. por lo que no fue considerado. En dos de los 10 casos de asma bronquial se registraron complicaciones, siendo estas, respectivamente, atelectasia crónica y neumatocele. En sjete ingresos por secuelas respiratorias de infecciones por adenovirus, la complicación fue síndrome bronquial obstructivo recurrente en seis e insuficiencia respiratoria crónica con dependencia por oxígeno en uno. Las complicaciones registradas en seis ingresos por malformaciones broncopulmonares fueron sindrome bronquial obstructivo recurrente en cuatro y neumonía a repetición en

\section{Tabla 1}

Hospitalizaciones por enfermedades respiratorias crónicas y recurrentes: Causas de ingreso según diagnostico principal

\begin{tabular}{|c|c|c|c|c|c|}
\hline \multirow[b]{2}{*}{ Diagnóstico principal } & \multicolumn{5}{|c|}{ Causas de ingreso } \\
\hline & Neumonía & $\begin{array}{l}\text { Crisis } \\
\text { obstr. }\end{array}$ & Otras* & $\mathbf{n}$ & $(\%)$ \\
\hline S. bronquial obstr. recum. & 120 & 44 & 4 & 168 & $(69,4)$ \\
\hline Neunopatías a repeticjón & 19 & 1 & 8 & 28 & $(11,6)$ \\
\hline Displasia broncopulınonar & 10 & 2 & 2 & 14 & $(5,8)$ \\
\hline Asing & 2 & 8 & - & 10 & $(4,1)$ \\
\hline Secuela adenovirus & 3 & 2 & 2 & 7 & $(2,9)$ \\
\hline Malformaciones broncopulmon. & l & 2 & 3 & 6 & $(2,5)$ \\
\hline Fjbrosis quística & 3 & 1 & 1 & 5 & $(2,1)$ \\
\hline Hemosiderosis pulmonar & 1 & 1 & 1 & 3 & $(1,2)$ \\
\hline Bronquitis recurte no obst. & - & - & 1 & I & $(0,4)$ \\
\hline Total & $159(65,7)$ & $61(25.2)$ & $22(9,1)$ & 242 & $(100,0)$ \\
\hline
\end{tabular}

* Atelectasia. hemoptisis, estudio enfermedad de base, medieión nocturna de saturación de hemoglobina con oxígeno. 


\section{Tabla 2}

Enfermedades asociadas segûn diagnóstico principal

\begin{tabular}{|c|c|c|c|c|c|c|}
\hline \multirow[b]{3}{*}{ Enfermedad asociada } & \multicolumn{6}{|c|}{ Diagnóstico principal } \\
\hline & \multicolumn{2}{|c|}{$\begin{array}{l}\text { S. b. obst. } \\
\text { recurrente } \\
\text { n: } 168\end{array}$} & \multicolumn{2}{|c|}{$\begin{array}{l}\text { Neumopatía } \\
\text { repetida } \\
\text { n: } 28\end{array}$} & \multicolumn{2}{|c|}{$\begin{array}{l}\text { E.respir. } \\
\text { crónica } \\
\text { n: } 46\end{array}$} \\
\hline & $\mathbf{n}$ & $(\%)$ & $\mathbf{n}$ & $(\%)$ & $\mathbf{n}$ & $(\mathscr{\%})$ \\
\hline Enfermedad neurológica & 29 & $(7,2)$ & 20 & $(71,4)$ & 6 & $(3,3)$ \\
\hline Sindrome de Dow口 & 19 & $(11,3)$ & - & & - & \\
\hline Reffujo gastroesofásico & 7 & (4.2) & 2 & $(7,2)$ & 19 & $(42,2)$ \\
\hline Deficiencia inmunidad & - & & 6 & $(21,4)$ & - & \\
\hline Otras & - & & - & & 4 & $(8,9)$ \\
\hline \% E. esociada & & $(32,7)$ & & $(100)$ & & $(64,4)$ \\
\hline
\end{tabular}

S. b. obst.: sindrome bronquial obstructivo recurrente.

Orras: malfomación congénita cardiaca o desnutrición.

los otros dos. La obstrucción bronquial recurrente fue la complicación en las tres oportunidades en que ingresó un paciente con hemosiderosis pulmonar.

En la tabla 2 se puede comprobar que en la mayoría de los casos en que el diagnóstico principal era síndrome bronquial obstructivo recurrente no se registró una enfermedad asociada 0 extrarrespiratoria, pero cuando ello ocurrió se trataba con más frecuencia de alguna enfermedad neurológica. En contraste, cuando la afección principal era neumonía a repetición, todos los casos sufrían una enfermedad extrarrespiratoria asociada, siendo también esta, con mayor frecuencia, neurologica, y constituyendo al mismo tiempo la enfermedad de base. Finalmente, en las hospitalizaciones por enfermedades respiratorias crónicas, las enfermedades asociadas más destacadas fueron reflujo gastroesofágico y enfermedad neurológica (retardo desarrollo psicomotor), esta última especificamente en hospitalizaciones por displasia broncopulmonar.

La duración promedio de las 242 hospitalizaciones por enfermedades respiratorias y recurrentes fue 11,39 días con amplios márgenes de variación (de 1 hasta 125 días) en contraste con 8,8 días en el total de los ingresos al Servicio de Pediatría. Se registraron 24 casos de hospitalizaciones prolongadas (definidas como de más de 20 días de duración), casi una por cada diez ingresos evaluados. Las enfermedades respiratorias de base más frecuentes en esta situación fueron también el síndrome bronquial obstructivo recurrente ( $n=9$; duración 20 a 58 días) y la neumonía a repetición $(\mathrm{n}=9$; duración 25 a 125 días. Siguen en orden de frecuencia las enfermedades respiratorias crónicas: fibrosis quística ( $\mathbf{n}=1$; duración 95 dias) , displasia broncopulmonar ( $n=2$; duración 44 y 73 dfas), asma ( $\mathrm{n}=1$; duración 27 días), malformaciones broncopulmonares (n 2 ; duración 20 y 28 dras). En todas las hospitalizaciones prolongadas había una enfermedad extrarrespiratoria, de base 0 asociada: neurologica en 13 casos $(54 \%)$, reflujo gastroesofágico en 5 ingresos (23\%), en tres inmunodeficiencia y síndrome de Down en el restante. Las hospitalizaciones de mayor duracion correspondieron a displasia broncopulmonar y neumonía repetida en pacientes con enfermedad de base no respiratoria.

En 33 de 143 pacientes $(21,7 \%)$ se registraron tres o más ingresos en un año, $24(77,4 \%)$ tenían síndrome bronquial obstructivo, 5 (16,1\%) enfermedades respiratorias crónicas (displasia broncopulmonar, asma, hemosiderosis pulmonar, secuela de adenovirus) y 2 $(6,5 \%)$ neumonías a repetición. Veintitrés de estos casos tenían enfermedades no respiratorias asociadas $(74,2 \%), 8$ sufrían afecciones neuro- 
lógicas $(25,8 \%) ; 6$ tenían reflujo gastroesofágico $(19,4 \%)$ y 3 síndrome de Down $3(9,6 \%)$. Otros diagnósticos observados en 6 casos con ingresos reiterados correspondían a inmunodeficiencia, incoordinación faríngea con reflujo gastroesofágico, afecciones presuntamente genéticas, desnutrición, anemia, obesidad.

De un total de 22994 d́as-cama ocupados en el Servicio de Pediatría durante el período. $2893(12,5 \%)$ correspondieron a los 242 ingresos incluidos en este análisis. Si se extrapola el promedio de días de estada a los 322 ingresos efectivos de enfermos respiratorios crónicos o recurrentes, resultaría una cifra de 3849 díascama o 16,7\% del total del servicio. El coste estimado del día-cama en el hospital fue -en la misma época- aproximadamente de $\$ 40000$ en moneda chilena (m./n.) (US\$100), por lo que el gasto originado por este concepto puede estimarse en $\$ 15400000 \mathrm{~m} / \mathrm{n}$. (US\$ 8500 ).

Los exámenes más frecuentes fueron los requeridos para guiar el tratamiento de la enfermedad que causó el ingreso. Se realizaron 621 determinaciones de gases en sangre arterial (2,56 por ingreso), 446 de electrólitos plasmáticos (1,84 por ingreso), 442 radiografías de tórax ( 1,82 por ingreso), 334 mediciones de proteína $C$ reactiva en el plasma (1,38 por ingreso), 134 hemocultivos $(0,55$ por ingreso) y 114 reacciones de inmunofluorescencia para antígenos virales en las secreciones respiratorias $(0,47$ por ingreso). Con menos frecuencia se hicieron exámenes para el estudio de la enfermedad de base. como concentración sérica de inmunoglobulinas, electrólitos en sudor, entre otros. Los exámenes de mayor costo registrados en esta serie fueron 11 tomografías axiales computadorizadas, ocho procedimientos de fibrobroncoscopía, siete cintigrafías de ventilación y perfusión, seis estudios ultrasonográficos, una cintigrafía esofágica y una angiografía pulmonar. El laboratorio central del hospital procesó 5243 examenes en las 242 hospitalizaciones de la muestra o 21,6 por cada egreso, dentro de un total, para el servicio de pediatría, de 57193 o 19,7 exámenes por alta. En términos de arancel 1 del FONASA el coste de los exámenes de los 242 ingresos analizados puede estimarse en $\$ 7220570 \mathrm{~m} . / \mathrm{n}$. (aproximadamente US $\$ 18000$ ) que, extrapolados a los 322 ingresos reales resultan en un costo estimado -con el criterio senalado- de $\$ 9607535 \mathrm{~m} . / \mathrm{n}$., o $15,3 \%$ del costo total de los examenes del servicio de pediatría, que ascendió a \$ 62735336 (US\$157000), sin contar el valor de otros exámenes 110 realizados en ese laboratorio, como las a ariografías de torax, tomografías axiales, broncoscupías, ultrasonografías, que para los pacientes $c^{l}$ la muestra alcanzaron a 625 exámenes "especiales" (aproximadamente 2,6 por ingreso) y' fue estimado en otros \$5 $338240 \mathrm{~m} / \mathrm{n}$. (US\$133.6) según el arancel ya mencionado. Los costos de otros exámenes realizados para el estudio de las intercurrencias intrahospitalarias no fueron valorizados monetariamente.

En lo que respecta a los tratamientos más frecuentemente empleados, en 221 (92\%) hospitalizaciones se usaron broncodilatadores por una extension media de 10,9 días; oxígeno en 188 $(77,7 \%)$ por 9,3 días en promedio; antibióticos en 134 ingresos $(55,4 \%)$ : más de uno en $77 \mathrm{ca}$ sos (31,8\%) por una media de 12 días o s6lo uno en $57(23,5 \%)$ duración promedio, 6,5 días; corticoides orales, endovenosos o inhalatorios, se emplearon en 165 admisiones $(68 \%)$ con duración media de 2,6 días para los intravenosos y 5,6 días en los orales. En $202(83,5 \%)$ hospitalizaciones se emplé kinesjterapia respiratoria por uná extensión media de 11,1 días.

\section{Comentario}

Las enfermedades respiratorias crónicas o recurrentes representaron más de una de cada cinco hospitalizaciones por enfermedades respiratorias durante el año 1994 en el Servicio de Pediatría Hospital San Borja-Arriarán e implican costes proporcionalmente mayores por días, cama y consumo de examenes de laboratorio que el promedio global del Servicio de Pediatría, a pesar que en la mayor parte de los casos no se trata de ingresos electivos, sino de emergencias que requieren manejo de nivel terciario por una descompensacion o recurrencia. Sólo ocasionalmente se trata de pacientes admitidos para evaluación por enfermedades de difícil diagnóstico o que requieran procedimientos que exigen hospitalizacion.

En los pacientes de esta serie se pueden distinguir tres conjuntos, de acuerdo a las características clínicas: unos tienen afecciones respiratorias con síntomas recurrentes, con escaso o ningún sustrato estructural o secuelas anátomi- 
cas y funcionales; otros una enfermedad broncopulmonar crónica de base; finalmente algunos tienen un trastorno de base no respiratorio, pero con una afección broncopulmonar secundaria resultante, que es la causa de las admisiones al hospital.

Los pacientes de enfermedades respiratorias con sintomas recurrentes corresponden principalmente a los que egresan con diagnostico de síndrome bronquial obstructivo recurrente, que en la mayor proporción es -presuntamente- secundario a infecciones virales. cuyos síntomas persisten durante algún tiempo, especialmente en el primer año de vida, en el que sufren varias reagudizaciones. En la mayoría de estos casos no se registran complicaciones de la enfermedad de base ni enfermedades extrarrespiratorias asociadas. Ellos dan cuenta del mayor número de hospitalizaciones de la muestra, pero sus evoluciones clínicas son menos graveg que en los dos grupos siguientes y demandan menor consumo de recursos. Son pocos, entre estos niños, los que cursan con hospitalizaciones prolongadas o tórpidas, lo que, por lo general ocurre en los que, además, tienen otras enfermedades asociadas (preferentemente neurológicas).

Los pacientes con enfermedades respiratorias crónicas constituyen la segunda agrupación entre las hospitalizaciones de esta muestra. Su evolución es más compleja y prolongada, requiriendo para su manejo mayores recursos que la mayoría de los pacientes del primer grupo referido.

En el tercer lugar se reúne una heterogénea varjedad de pacientes cuya enfermedad de base es extrarrespiratoria. más frecuentemente neurológica o con algún tipo de deficiencia de la inmunidad, a las que se agrega como complicación una afección respiratoria, que pronto se transforma en el principal motivo de ingreso al hospital, con evoluciones torpidas y graves, complicadas por neumopatías, secuelas de bronquiectasias, insuficiencia respiratoria crónica con dependencia del oxígeno, hospitalizaciones reiteradas y prolongadas. Estos pacientes demandan al hospital mayor consumo de recursos que los dos grupos precedentes, con menor rendimiento a largo plazo, y corresponden, en su mayoría, a los que egresan con diagnostico de "neumopatías a repetición". Su manejo ambulatorio en el curso de los últimos 10 años ha mejorado, sin embargo pudiese mejorar más toda- vía, reduciendo el riesgo de hospitalización, optimizando los programas para pacientes crónicos ambulatorios y dotando a estos con recursos apropiados de estudio, tratamiento, educación y apoyo técnico a la familia en los aspectos pertinentes.

La alta frecuencia de diagnósticos sindromáticos probablemente guarda relación con la precocidad -en la vida - con que ocurren estas hospitalizaciones: cuando está comenzando a manifestarse la enfermedad básica o aún no ha concluido el estudio ambulatorio que permita identificarla con mayor precisión, ya que más la mitad de los casos son menores de un año edad y sobre dos tercios menores de dos años.

La estimación de costes por exámenes de laboratorio en un hospital moderno, dadas las elevadas sumas de dinero involucradas, en especial cuando se trata de hospitales públicos en cuya operación está comprometido el patrimonio de todos los contribuyentes y los derechos a la atención de salud de los menos favoreciơos, debería incluir como mínimo los implícitos en estructura, equipos, personal, reactivos, amortizaciones, mantencion, procesamiento, información, controles de calidad. Lo mismo vale para el cálculo de todos los otros aspectos de la atención médica. Como en el medio en que se desarrolla este análisis no se cuenta con información más precisa sobre estas materias, se eligió un arancel estándar, que subestima los costos reales y sólo permite algunas comparaciones muy gruesas en las proporciones de los gastos en la atención entre los pacientes estudiados y el conjunto de los niños atendidos en el hospital, las que muestran que algo más de un décimo de las hospitalizaciones genera un sexto de los gastos de laboratorio. Muchas causas de ingreso por enfermedades respiratorias crónicas o recurrentes de las identificadas en este trabajo pudiesen ser controladas con mejor manejo ambiental, terapia ambulatoria agresiva y precoz y medidas generales de rehabilitación y prevención, como ha ocurrido, por ejemplo, con la fibrosis quística en otros países ${ }^{8}$. Tal pudiese ser el caso en enfermos neurológicos, con síndrome de Down o con reflujo gastroesofágico, trastornos relacionados con 102 de las 242 admisiones revisadas. Parece razonable suponer que estos pacientes, como también los que sufren enfermedades obstructivas recurrentes, se beneficiarian mejorando su manejo ambulatorio con medidas para 
aumentar la capacidad resolutiva de los médicos de atención primaria mediante programas apropiados de capacitación; poner a disposición de las unidades primarias, recursos profesionales y lécnicos para realizar en el lugar estudios básicos, dar el tratamiento ambulatorio necesario en forma oportuna y hacer un seguimiento adecuado de los pacientes; optimizar la coordinación entre los policlínicos de especialidades del nivel secundario (respiratorio, neurológico, inmunológico, medicina física y rehabilitación, kinesiterapla, nutrición) y los primarios. Con las medidas señaladas sería probablemente posible abreviar la evolución, anticiparse a las complicaciones y reducir el riesgo de hospitalización. También conveniente disponer en los hospitales de los recursos que faciliten los trámites de estudio y tratamiento de aquellos pacientes que, ineludiblemente, deban ser ingresados para reducir los riesgos y costes de permanencia en el hospital.

\section{Referencias}

1. Dfaz A: Neumonias crónicas o recidivantes. Pediatría (Santiago) 1993; 36: 151-156.

2. Warren $E$, Rezelmann $M$ : Diagnosing the cause of recurrenl and persistent pnemmonia in children. Pediatric Annals 1993:22; $561-568$.

3. De Baets $F$, Pauwets $R$. Schramme I, Leroy J: IgG subclass specific antibody response in recurrent bronchitis. Arch Dis Child 1991: 66: 1378-1382.

4. Greenough A. Maconochie I. Yukse? B: Recurrent respiratory symptoms in the first year of life following preterm delivery. J Perinat Med 1990; 18; 489-494.

5. Van Bever $H$ : Definitions of chronic respiratory disease what about children. Eur Resp J 1990; 3: 1096-] 097 .

6. Herrera $O$, Fielbaum $O$ : Sfndrome bronquial obstructivo del lactante. En: Herrera O. Fielbaum O (ed): Manual de enfermedades respiratorias infantiles. Ed. Mediterráneo, Santiago, Chile, 1995; 221-228.

7. Mertsola J, Ziegler T, Ruuskanen O, Vauto T, Koivikko A. Halonen $P$ : Recurrent wheezy bronchitis and viral respiratory infections. Arch Dis Child 1991; 66: 124-129.

8. Corey M, Mac Laughlin FG. Levin HA: A comparisson of survival, growth and pulmonary function in patients with cystic fibrosis in Boston and Toronto. J Clin Epidemiol 1988; 41:583-591. 